\title{
MiR-513a-5p Aggravates Atherosclerosis Phenotype by Downregulating TFPI2 in
}

Vitro

Chun Wang, Feng Yao

This manuscript has been withdrawn by the authors as it was submitted and made public without the full consent of all the authors. Therefore, the authors do not wish this work to be cited as reference for the project. If you have any questions, please contact the corresponding author. 